\title{
Designing trials of complex interventions for efficacy and mechanisms evaluation
}

\author{
Richard A Emsley ${ }^{1 *}$, Jonathan Green², Graham Dunn \\ From Clinical Trials Methodology Conference 2011 \\ Bristol, UK. 4-5 October 2011
}

\section{Objectives}

There have been recent developments in statistical methods for assessing the mechanisms through which a complex intervention acts, with particular applications in mental health trials. This literature has focused on causal mediation analysis, which differs from statistical mediation analysis by its explicit acknowledgement and exposition of the necessary assumptions, particularly that of no unmeasured confounding between the putative mediators or mechanisms and outcomes. Instrumental variable estimation procedures can produce valid causal estimates even in the presence of this unmeasured confounding, but the problem then becomes finding valid instruments to identify the statistical models. So far, however, little attention has been given to the issue of designing trials of complex interventions with this analysis in mind.

\section{Methods}

We introduce the instrumental variables estimation procedure, and discuss some possible methods for obtaining instruments. We then propose four alternative designs for trials of complex interventions in mental health which could produce these potential instruments:

1. "Waiting list control", where the control group receive the intervention after a specified period of time;

2. "Innocuous vaccine", where all participants are measured on a baseline variable strongly related to the putative mechanism;

3. "Parallel trials", where a concurrent set of trials are run in parallel with different interventions but common measures;

\footnotetext{
* Correspondence: richard.emsley@manchester.ac.uk

${ }^{1}$ Health Sciences Research Group, School of Community Based Medicine,

The University of Manchester, Manchester, UK

Full list of author information is available at the end of the article
}

4. "Mediated moderation", where a potential instrumental variable is included as a baseline measure.

\section{Results}

We demonstrate how these trial designs can help validate the causal mediation analysis and strengthen some of the fundamental assumptions underpinning it. We give examples with illustration of the "parallel trials" design in a randomised trial of cognitive behaviour therapy in psychosis, and a "mediated moderation" design in parent mediated treatment trial in child psychiatry.

\section{Conclusions}

Well-designed complex intervention trials should not only consider evaluating the efficacy of the intervention but also the putative mechanisms through which it acts. Adopting a suitable design for the trial both enables and strengthens the assessment of this mechanistic analysis.

\section{Acknowledgements}

All authors are investigators on an MRC methodology grant entitled "Designs and analysis for the evaluation and validation of social and psychological markers in randomised trials of complex interventions in mental health" (G0900678). RE is supported by an MRC Career Development Award in Biostatistics (G0802418). RE and GD are members of the UK Mental Health Research Network Methodology Research Group.

\section{Author details}

${ }^{1}$ Health Sciences Research Group, School of Community Based Medicine, The University of Manchester, Manchester, UK. ${ }^{2}$ Mental Health and Neurodegeneration Research Group, School of Community Based Medicine, The University of Manchester, Manchester, UK.

Published: 13 December 2011

doi:10.1186/1745-6215-12-S1-A143

Cite this article as: Emsley et al:: Designing trials of complex interventions for efficacy and mechanisms evaluation. Trials 201112 (Suppl 1):A143.
C Biomed Central

C 2011 Emsley et al; licensee BioMed Central Ltd. This is an open access article distributed under the terms of the Creative Commons Attribution License (http://creativecommons.org/licenses/by/2.0), which permits unrestricted use, distribution, and reproduction in any medium, provided the original work is properly cited. 